\title{
LIII. Photographs of Jupiter taken at the Lowell observatory, April 1909, by Mr. E. C. Slipher and the Director, Dr. Percival Lowell
}

Dr. Lowell

To cite this article: Dr. Lowell (1910) LIII. Photographs of Jupiter taken at the Lowell observatory, April 1909, by Mr. E. C. Slipher and the Director, Dr. Percival Lowell, Philosophical Magazine Series 6, 19:112, 488-497, DOI: 10.1080/14786440408636828

To link to this article: http://dx.doi.org/10.1080/14786440408636828

曲 Published online: 21 Apr 2009.

Submit your article to this journal $\pi$

Џll Article views: 3

Q View related articles $₫$ 
other by momenta, is valid apart from any question of ignored coordinates. Thus generally the force-equations being

$$
\mathrm{F}\left(x_{p}\right)=\frac{d}{d t} \frac{\partial \mathrm{L}}{\partial x_{p}}-\frac{\partial \mathrm{L}}{\partial r_{p}}, \quad \mathrm{~F}\left(x_{m+r}\right)=\frac{d \xi_{m+r}}{d t}-\frac{\partial \mathrm{L}}{\partial x_{m+r}},
$$

and $\dot{x}_{m+r}$ being $-\frac{\partial \mathrm{L}}{\partial \xi_{m+r}}$, there is exact agreement between these forces and those derived from the formula

$$
\frac{d}{d t} \frac{\partial \mathrm{T}_{0}}{\partial \dot{x}}-\frac{\partial \mathrm{T}_{0}}{\partial x}
$$

When the coordinates $x_{m+r}$ do not appear in the coefficients of $\mathrm{T}_{0}$, and there is no corresponding external force, $\frac{d \xi_{m+r}}{d t}=0$, $i$. e. these momenta are constant. Thus what for the general case is an admissible modification of the expression for energy, and of the form of the force-equations, becomes in the case of ignorable coordinates the reduced form of the system. In this case $K$ has the true character of a potential energy, while the existence of $I$ marks the interaction between the two groups originally shown by the presence of product terms of the form $\dot{x}_{p} \dot{x}_{m+r}$

In conclusion it may be noted that $\mathrm{T}$ is the minimum value of $\mathrm{T}_{0}$ which can be obtained by giving real values to $\dot{x}_{m+1} \ldots \dot{x}_{n}$, because the minimum problem leads to the group of equations (4) with $\xi_{m+r}=0$.

LIII. Photographs of Jupiter taken at the Lowell Observatory, A pril 1909, by Mr. E. C. Sllipher and the Director, Dr. Percival Lowell.' By Dr. LoweLL*.

THE photographs of Jupiter taken here this spring may 1 be considered under two distinct heads :-1, researches on what measures of them yield ; 2 , study of the markings represented.

The first investigation was rendered possible by the great size to which it proved the images could be enlarged without loss of detail. The results are interesting, for they have something to say about the planet's physical condition.

1. We will begin with the ellipticity of Jupiter's globe. Measures of the equatorial and polar diameters in five

* Communicated by the Author. 
images on a plate of mine taken April 14 gave as a mean for the ratio of the two :-

$$
\begin{gathered}
\frac{\text { polar diameter }}{\text { equatorial diameter }}=\frac{1}{1 \cdot 0677} . \\
\text { This makes the oblateness }=\frac{a-b}{a}=\frac{1}{15 \cdot 8}, \\
\text { and for the ellipticity }=\frac{a-b}{b}=\frac{1}{14 \cdot 8} .
\end{gathered}
$$

At the time of the photographs the phase amounted to $\frac{1}{200}$ of the equatorial diameter. This was partially offset by the irradiation from the limb. Also the poles of the planet are notably darker than the rest of the disk, causing a less visible extension there, and this effect is increased by the photographic plate. As the corrections thus work opposite ways we may, to a sufficient degree of approximation, consider them as neutralizing one another. And in this connexion a caveat should be entered against measures made with a colour screen by daylight, especially when the planet shows a phase, unless these are subsequently corrected for relative irradiation and loss by faintness on the terminator. For in such cases measures may show less than reality, thus erring in the opposite direction from what the earlier measures, where irradiation was neglected, did.

We will now compare this photographic value with what micrometric and heliometer measures of the planet have yielded.

From the various determinations which have been made

\begin{tabular}{|c|c|c|c|}
\hline J. D. Cassini $\quad \ldots \ldots \ldots$ & $\begin{array}{l}\text { Date. } \\
1691\end{array}$ & $\begin{array}{l}\text { Oblateness. } \\
1: 15\end{array}$ & $\begin{array}{l}\text { Interesting as being } \\
\text { the first ever made. }\end{array}$ \\
\hline Bessel ...... & 1833 & $1: 15 \cdot 73$ & $\begin{array}{l}\text { Heliometer cor- } \\
\text { rected for phase. }\end{array}$ \\
\hline Secchi & 1856 & $1: 16 \cdot 06$ & Micrometer. \\
\hline Kaiser & 1857 & $1: 15 \cdot 62$ & 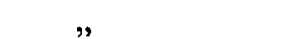 \\
\hline Lohse & 1873 & $1: 15 \cdot 80$ & " \\
\hline (Hough ..... & 1880 & $1: 16 \cdot 76)$ & \\
\hline Barnard $\ldots \ldots \ldots \ldots$ & $1892-4$ & $1: 15 \cdot 99$ & $"$ \\
\hline $\begin{array}{l}\text { W. H. Pickering, Lowell } \\
\text { Obs. }\end{array}$ & 1894 & $1: 16 \cdot 11$ & $"$ \\
\hline $\begin{array}{c}\text { (Lewis } \quad \ldots \ldots \ldots \ldots \ldots \ldots \\
\text { See } \ldots \ldots \ldots \ldots \ldots \ldots \ldots\end{array}$ & $\begin{array}{l}1894-5 \\
1900\end{array}$ & $1: 16 \cdot 7)$ & \\
\hline See $\ldots \ldots \ldots \ldots \ldots \ldots$ & 1900 & $1: 15 \cdot 53$ & $"$ \\
\hline
\end{tabular}
of Jupiter's oblateness, we may perhaps select the following as the best:- 
From the motion of the perijove of the $V$ th satellite have been deduced:-

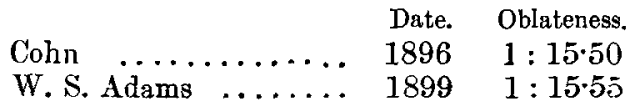

For some reason the oblateness deduced from measures of the orbits of the satellites seems to conie out too large. Thus La Place got from those of the other satellites $\frac{1}{14 \cdot 39}$ and De Damoiseau $\frac{1}{13 \cdot 44^{\circ}}$. The oblateness has now been so well measured that we are certain that these denominators are too small.

We see, then, that the mean of the visual measures and of the photographic agree thus :-

$$
\begin{array}{r}
\text { Oblateness, mean of visual determinations . } \frac{1}{15 \cdot 83}, \\
" \quad, \quad \text { photographic determinations } \frac{1}{15 \cdot 8} .
\end{array}
$$

A concordance so striking shows how serviceable these photographs may be for future measures of the rotation periods of different parts of the disk. For the present ones can be compared with those taken at any later date.

The actual ellipticity of Jupiter combined with its rate of rotation gives us an insight into the planet's present physical condition.

By Clairaut's theorem the limits between which the ellipticity of a rotating body must lie, no matter what the law of its distribution of density be, are $\frac{1}{2} \phi$ and $\frac{5}{4} \phi$ where $\phi$ is the ratio of the centrifugal force to gravity at its equator when the body is homogeneous. Gravity is the attraction there less the action of the centrifugal force, therefore :

$$
\phi=\frac{\omega^{2} a}{g}=\frac{\omega^{2} a}{f-\omega^{2} a}=\frac{1}{\frac{1^{y} f}{a}-1}
$$

where

$$
\begin{aligned}
& \omega=\text { angular rate of rotation, } \\
& \mathrm{T}=\text { its period, } \\
& a=\text { the equatorial radius, } \\
& g=\text { gravity, } \\
& f=\text { the attraction of the body on a point at its } \\
& \quad \text { equator. }
\end{aligned}
$$

The limit $\frac{1}{2} \phi$, as the equation by which it is determined shows, corresponds to a distribution of density where all the 
matter composing the body is concentrated at its centre; the limit $\frac{5}{4} \phi$, to that in which the body is homogeneous.

Now, if we know the body's equatorial radius and its ellipticity, we can determine its volume, and from its mass its mean density; and if in addition we know its rate of rotation, we can from its density and its rotation rate deduce $\phi^{\prime}$ by the formula

$$
\mathrm{V}^{\prime}=\mathrm{V} \frac{\mathrm{T}^{2}}{\overline{\mathrm{T}}^{\prime} \overline{2}} \frac{\rho}{\rho^{\prime}}
$$

where the unaccented quantities refer to the Earth and the accented to Jupiter.

For $V^{\prime}=\frac{\omega^{\prime 2}}{k 4 \pi \rho^{\prime}}$ may thus be found, $\mathrm{V}$ being 0.00115 , and $k$ being the constant of attraction ; and $\phi^{\prime}$ may be expressed in terms of $\mathrm{V}^{\prime}$ by a process which the reader will find given in 'Tisserand's Mecanique Celeste.

Tisserand's data for the densities \&c. are not in accordance with modern values. It is necessary, therefore, to recalculate $\phi$. Using, now, for Jupiter the most modern determination of the equatorial radius and the ellipticity which, as we have seen, the measures of the photographs bear out, to wit :-

$$
\begin{aligned}
a & =38^{\prime \prime} \text { at Jupiter's mean distance, the solar parallax } \\
& \text { at } 8^{\prime \prime} \cdot 80, \\
& =89040 \text { miles or } 143300 \mathrm{~km} . \\
\mathrm{T} & =9^{\mathrm{t} .} 842 \text { at Jupiter's equator, }
\end{aligned}
$$

and $\epsilon=\frac{1}{14 \cdot 8}$ or $\eta=\frac{1}{15 \cdot 8}$,

where $\epsilon=$ the ellipticity $\frac{a-b}{b}$ and $\eta$ the oblateness $\frac{a-b}{a}$,

we find

$$
\phi^{\prime}=\frac{1}{10 \cdot 2} \text {. }
$$

The ellipticity is the more philosophic quantity to use, although the oblateness is more commonly employed, because the ellipticity associates itself directly with $\phi^{\prime}$, and thus with the planet's physical state.

The limits then between which the ellipticity could lie are $\frac{1}{20 \cdot 2}$ and $\frac{1}{8 \cdot 1}$, the former corresponding to total concentration at the centre, the latter to homogeneity*. We thus

* For a new and more exact method of determining these linits and $\phi$, see paper by the avthor appearing in a following number. 
see that Jupiter with an ellipticity of $\frac{1}{14 \cdot 8}$ fulfils Clairaut's law, and furthermore that the matter composing him comes nearer to central condensation than to homogeneity.

Going into this more carefully and comparing the corresponding data for the Earth, Mars, and Saturn, we get the following table :-

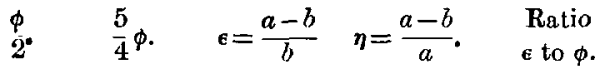

$$
\begin{aligned}
& \text { Earth ..... } \frac{1}{577} \quad \frac{1}{231} \quad \frac{1}{295} \quad \frac{1}{296}=0.975 \\
& \text { Mars } \ldots \ldots \frac{1}{435} \quad \frac{1}{174} \quad \frac{1}{189} \quad \frac{1}{190} \quad=1 \cdot 151 \\
& \text { Jupiter } \ldots \frac{1}{20 \cdot 3} \quad \frac{1}{8 \cdot 1} \quad \frac{1}{14 \cdot 8} \quad \frac{1}{15 \cdot 8}=0.682 \\
& \text { Saturn } \ldots \quad \frac{1}{10} \quad \frac{1}{4} \quad \frac{1}{8 \cdot 7} \quad \frac{1}{9 \cdot 7}=0.575
\end{aligned}
$$

These are the only planets for which we have the complete data. Mercury and Venus rotate so slowly, in 88 and 225 days respectively, that their oblateness is too small to be measured, so that nothing can be deduced of their laws of density. Of Uranus and Neptune we lack as yet the rotation periods, and in the case of the last even the flattening, so that their distribution of matter remains equally hid.

In considering the table we see that the Earth and Mars lie nearer homogeneity than central condensation, since the conditions for these states are $\epsilon=0.5 \phi$ and $\epsilon=1.25 \phi$. Jupiter and Saturn, on the other hand, approach central condensation, Saturn indeed almost reaching it.

Now, when we reflect upon the great pressures to which the matter composing the major planets must be subjected owing to their enormous masses, we perceive that such slight densities as they present can only be maintained through great internal heat. The force of attraction at Jupiter's equator, for instance, would be $2 \cdot 61$ times the Earth's were his substance homogeneous, and 2.51 times were all his matter concentrated at his centre, being given by

$$
f=k \frac{4 \pi \rho b}{3}\left(1+\frac{3}{5} \epsilon-\frac{9}{35} \epsilon^{2}+\text { etc. }\right),
$$

where $b$ is the polar radius, in the one case, and by

$$
f=k \frac{m}{a^{2}}
$$

in the other. 
As we have seen that he more nearly approaches the central concentrated than the homogeneous state we may take 2.54 as the value. His centrifugal force, so called, at his equator is

$$
\frac{a^{\prime}}{a} \frac{\mathrm{T}^{2}}{\mathrm{~T}^{\prime 2}} \frac{1}{289} \text {, or } \quad \cdot 23 \text { times the Earth's attraction, }
$$

whence we finally get

2.31. for the force of gravity at his equator compared with that at the Earth's.

If Jupiter were cold this would cause a density greatly exceeding the Earth's, while the facts are the other way, Jupiter's mean density being to the Earth's as 1.32 to 5.527 . It is evident, therefore, that he must be expanded by heat.

Furthermore, the gradation in density from core to cuticle must be gradual. There can be no sudden change from a dense kernel of Jupiter to a vast gaseous husk. For the great pressure in such a case would act to thin the air envelope relatively more than in the case on Earth. Nor would any permissible increase in the relative amount of air held by the planet come within the figures needed. For if the density of the nucleus were only that of the Earth, a modest estimate, the volume of the air outside it would be about 9 times its own, an amount of air with which we have no warrant for crediting it.

2. The next point is the position of the several belts. The measures of the images on the plates of April 14 give for their apparent places, taking the polar radius as the radius of the sphere of comparison, which, since the tilt was only $1^{\circ} \cdot 3$, may be taken as the apparent minor axis of the ellipse :-

Antarctic belt . . . . . . . . . $-37^{\circ} \cdot 2$

South Temperate belt . . . . . . . $-27^{\circ} \cdot 6$

South edge of S. Tropical belt . . . . $-19^{\circ} 5$ dark line in $\quad " \quad, \quad$. . . . . $\quad-15^{\circ} \cdot 9$

North edge of South Tropical belt . . . $-6^{\circ} 8$

North Tropic belt . . . . . . . . $+9^{\circ} \cdot 4$

North stripe of S. Tropical belt . . . +17.8

North Temperate belt . . . . . . . $+35^{\circ} 0$

At the time the photographs were taken, April 14, the latitude of the planet's equator with regard to the Earth was $-1^{0} \cdot 3$. 
Introducing this correction we have :

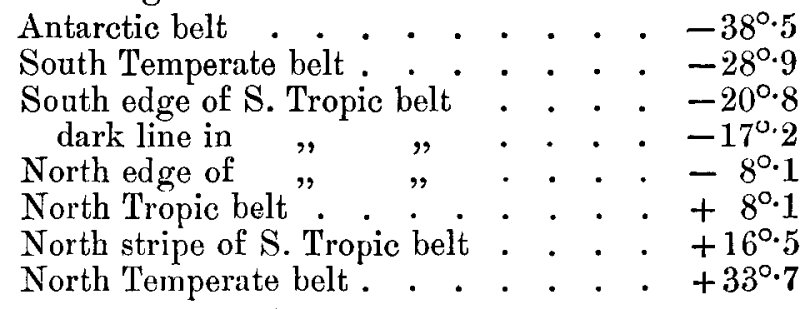

From this we see that the belts near the equator are here symmetrically disposed with regard to that line and regardless of the declination of the Sun at the time. Hence they cannot be caused by him but by Jupiter himself. Jupiter's cloud envelope is therefore totally unlike our own, and is a self-raised, not a sun-raised phenomenon. This state of things can only be due to great internal heat setting up currents which are persistent in their main features for long intervals of time. At other longitudes their latitudes differ ; a feature, interesting as it is, into which I do not propose here to enter, and unaffecting apparently the present conclusion.

The latitudes we have deduced are those on a sphere of radius equal to the polar radius. To correct these to the apparent latitude on the planet we proceed as follows. In an oblate spheroid any meridianal plane cuts out an ellipse in which all lines perpendicular to the polar axis are increased over those of the sphere with that axis as diameter in the proportion of $\frac{a}{b}$ to $b$ where

$$
\begin{aligned}
& a=\text { equatorial diameter } \\
& b=\text { polar }
\end{aligned}
$$

If, then, $\quad \alpha=$ colatitude measured on the sphere,

$$
\alpha^{\prime}=\quad, \quad, \quad \text { ellipse, }
$$

we have $\quad \tan \alpha^{\prime}=\frac{a}{b} \tan \alpha$,

This gives for the apparent latitude on the planet for the several belts :-

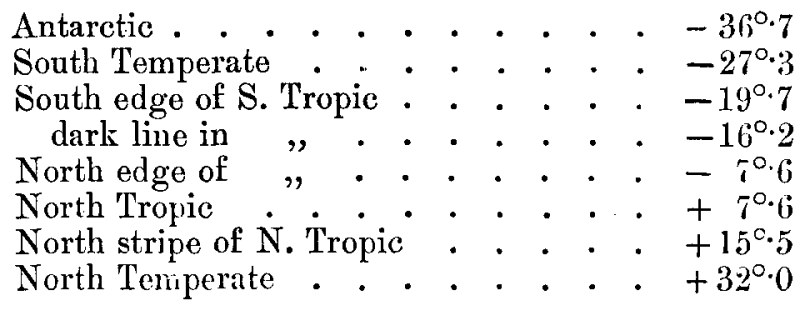


3. Turning now to the features themselves, we note that the great red spot, as a spot, practically no longer exists-it is now only faintly suffused with rose-but what was its oval cradle is well defined in the photographs, shown much as it has shown for the last thirty years. In fact in this connexion it is interesting to note that this cradle proves to be very much older than most people think. Long before the red spot made its sensational appearance the cradle it was to occupy was there. It is distinctly traceable in the drawings of Sir William Huggins made in 1859-1860, which he kindly sent me. My eye detected it the moment I examined them. Here then we have evidence of a feature which in its general outline has been stable and persistent for fifty years, a marvellous length of time for a cloud form as we know such things to continue to exist. This alone would suffice to demonstrate that Jupiter's meteorologic conditions owe nothing to the Sun. That this cradle then became the centre of a vast ruddy mass, which after a time disappeared to leave it in its former condition, indicated it as the seat of some violent outburst from below, over which the cloud veil, rent at the height of the explosion, settled down again, covering the furnace from sight. That the feature is so permanent hints at a certain plasticity as opposed to what we call gaseous in the constitution, and likens it to those permanent seats of disturbance with whose vents we are familiar on Earth. A volcano in embryo is what its behaviour points it out to be. It probably forms a connecting link between voleanoes on the one hand and sunspots on the other.

4. Perhaps the most interesting feature of the photographs is their exhibition of the wisps lacing the equatorial belt. These wisps were first detected and studied, I believe, by $\mathrm{Mr}$. Scriven Bolton, who communicated them here through Prof. Turner. 'They were then visually observed at Flagstaff, and many drawings made of them. Lastly, they appeared in the photographs, being well shown in the prints. They are very curious markings, being shreds or filaments of a dusky character traversing transversely the bright equatorial belt. They proceel always from black spots of a triangular shape set on the edge of the north or south tropical dark belts as the case may be. They join such to one another and to the medial dark line which with more or less interruption threads the planet's equator. Their course is usually inclined some forty-five degrees to the dark belts ; but sometimes they go straight across. When tilted they are as often right-handed as loft-handed. They are widest 
at their sides, where they leave the belts, and tenuous in mid-career. What they betoken is difficult to decide, because from their dark tint they would appear of a negative rather than of a positive character, gaps, that is, in the cloud envelop, rather than entities in themselves. That they give us a clue to the meteorological circulation of the planet is clear; but it is not at all clear what that clue is. That they are an essential feature of a planet in the early semichaotic state is indicated by a recent discovery at this observatory : that Saturn's belt is laced, though much more faintly, in precisely the same manner.

5. The colour of the dark markings of Jupiter is everywbere a cherry-red. As the physics of his condition show that he must be very hot, his density not being compatible with his gravity under any other supposition, the dark markings must be where the lower and hotter layers of the body show through. For that red heat is the maximum possible for his surface glow is evidenced by his albedo .78 of pure reflexion; no perceptible light being emitted by him. ${ }^{-} W_{\theta}$ are thus warranted in concluding that the lighter, yellowish belts lie at a higher altitude, and are what they seem to be, clouds. From Dr. Slipher's spectrograms these clouds appear to be made up in part at least of water-vapour, in part probably of other substance, though about this we bave no positive evidence, as the spectroscope informs us only that unknown gases are certainly present in the planet's atmosphere, but gives no assurance that these are condensed to cloud, possible and even probable as this may be. That the bright belts are cloud and show no self-emission of light appears also from their albedo being almost exactly that of cloud, $0 \cdot 78$ as against $0 \cdot 72$.

The various lines of evidence thus point to one conclusion : that Jupiter is still in a fiery chaotic state, that the materials that went to make him are still in disconnected fluid form, that they are much condensed toward his centre, but are kept from absolute solidification by his intense heat; in short, that he is in a midway stage between Sun and Earth.

6. To the great use to which these photographs and their successors may be put I may here draw attention. Permitting, as they do, of measurement of the planet's features it will be possible in the future to get a record of alterations in the appearance of the beits which no one may dispute and any one may verify by inspection himself of reprints from the negatives, and which in addition allow of quantitative valuation. Thus the rotation time of the several belts 
or of special details of them may be got with greater ease than at present, and any changes in rate from time to time may similarly be detected. Unaffected by any personal equation, the photographs have the further advantage of being original documents which can be used at first hand by any investigator.

Flagstaff, Arizona, Nov. 29, 1909.

LIV. On the Use of Mutual Inductometers. By Albert Campbell, B.A.*

(From the National Physical Laboratory.)

$\S 1$. Introductory.

$\S$ 2. Modified Mutual Inductance Bridge.

$\$ 3$. Measurement of Effective Inductance.

4. Null Method in Iron Testing.

§. Tests of Current Transformers.

\section{\$1. Introductory.}

T $\mathrm{N}$ a former paper $\dagger \mathrm{I}$ have described arrangements, which 1 we may call mutual inductance bridges, by which self inductances, even of very small amount, can be directly measured $\neq$. I wish here to discuss further these and other methods in which use is made of variable mutual inductances or, to give them the more convenient name, mutual inductometers.

\section{§2. Modified Mutual Inductance Bridge.}

In the equal arm bridge already discussed there is considerable loss of sensitivity resulting from the insertion of an auxiliary balancing self inductance in one of the arms. By a modification of the arrangement of the bridge, however, the use of the auxiliary coil is rendered unnecessary and the whole apparatus becomes simpler and more efficient. Let us first consider the more general case shown in fig. 1, where the ratio arms are not equal.

* Communicated by the Physical Society : read January 21, 1910.

+ Phil. Mag. Jan. 1908, p. 155.

$f$ I would mention here that many years ago Dr. Oliver Heaviside investigated a very general case of inductance bridges (Phil. Mag. p. 173, vol. xxiii. 1887). Most of the possible combinations are included in his paper.

Phil. Mag. S. 6. Vol. 19. No. 112. April 1910. 2 K 